\title{
Elastic Multi-Particle Systems for Bounded-Curvature Path Planning
}

\author{
Ali Ahmadzadeh, Ali Jadbabaie, George J. Pappas and Vijay Kumar
}

\begin{abstract}
This paper investigates a path planning algorithm for Dubins vehicles. Our approach is based on approximation of the trajectories of vehicles using sequence of waypoints and treating each way point as a moving particle in the space. We define interaction forces between the particles such that the resulting multi-particle system will be stable, moreover, the trajectories generated by the waypoints in the equilibria of the multi-particle system will satisfy all of the hard constraint such as bounded-curvature constraint and obstacle avoidance.
\end{abstract}

\section{INTRODUCTION}

The bounded-curvature path-planning problem, a central problem in robotics, involves planning a collision-free path for a nonholonomic robot moving amid obstacles, and has been widely studied (see, e.g., [2]). Dubins seminal work [3] characterizes shortest bounded-curvature paths in the absence of obstacles. He first notices that shortest boundedcurvature paths are made of arcs of minimum radius circles (C-segments) and straight line segments (S-segments). Using length reducing perturbations, Dubins shows that the structure of shortest paths, which is the sequence of (Csegments) and (S-segments) of which it is made, must be either CSC or CCC, where each segment may have zero length. Reif and Wang [4] show that finding the shortest bounded-curvature path amidst polygonal obstacles in the plane is NP-hard and. Fortune and Wilfong [5] show that the general feasibility algorithm is exponential in time and space. The NP-hardness result provide evidence that there are no efficient exact algorithms for curvature constrained path planning problem, and it justifies the approaches based on approximation and discretization used in most of the prior works on curvature-constraint path planning. Despite the NPhardness of the problem, there are efficient approximation algorithms [6], [7]. However, these algorithms are incomplete because they may not find a bounded-curvature path even if one exists.

In this paper we consider the problem of boundedcurvature path planning with a fixed length trajectory. The obstacles are modeled as union of disjoints discs. In this paper we present an approximate solution to this problem which can be generated in polynomial time, and we show that the algorithm is complete. Consider the trajectory of a vehicle as a fixed length bounded-curvature curve that connects the initial and final positions of the vehicle. We approximate this bounded-curvature curve by a polygonal curve, and each node of this polygonal curve represents a

Authors are with GRASP Laboratory, University of Pennsylvania, Philadelphia, PA 19104,USA

\{aliahmad, jadbabai, kumar, pappasg\} aseas.upenn.edu waypoint of the vehicle. First we find necessary and sufficient conditions for a bounded-curvature polygonal curve, then we construct the polygonal curve by treating each node as a moving particle in the space. We define interaction forces between the particles such that the resulting multiparticle system will be stable, moreover; the polygonal curve generated by the waypoints in the equilibria of the multiparticle system will satisfy all of the bounded-curvature necessary conditions as well as other hard constraints such as obstacle avoidance.

The paper is organized as follows: First, we find the conditions for bounded-curvature polygonal curves. Then, we try to solve the fixed length bounded-curvature path planning problem in the absence of obstacles. Next, we add obstacles to our model.

\section{Polygonal Curve Approximation}

In the rest of the paper, we assume that the trajectory of each vehicle is a fixed length bounded-curvature curve with a given initial and final configuration. Our approach is based on discrete approximation of a continuous curve using finite number of vertices.

Consider a bounded-curvature curve $\gamma$ with length $L$, connecting points $P$ and $Q$. We can approximate the curve using finite number of vertices each lying on the curve, connected by straight edges. The resulting polygonal curve $\gamma_{p}$ is represented by its ordered vertices $p_{0}, p_{1}, \ldots, p_{n} \in \mathbb{R}^{2}$, where $p_{0}=P, p_{n}=Q$ and $p_{i} p_{i+1}$ is the line segment connecting $p_{i}$ to $p_{i+1}$. The length of polygonal curve $\gamma_{p}$ is given by

$$
\operatorname{len}\left(\gamma_{p}\right)=\sum_{i}^{n}\left\|p_{i}-p_{i-1}\right\| \simeq L .
$$

With the assumption of equidistance edges with length $d$ we get

$$
d=\left\|p_{i}-p_{i-1}\right\| \simeq L / n .
$$

Assuming that $d \ll \frac{1}{\kappa}$, where $\kappa$ is the curvature bound, we can use the circle passing through the points $p_{i-1}, p_{i}$ and $p_{i+1}$, as an approximation to the osculating circle to the curve at $p_{i}$ (Fig. 1). Then the inverse value of the radius $r_{i}$ is an approximation of the curvature at $p_{i}$. Let $A$ denotes the area of the triangle $p_{i-1} p_{i} p_{i+1}$. and $d_{i j}=\left\|p_{i}-p_{j}\right\|$. The discrete curvature $\kappa_{i}$ is given by

$$
\kappa_{i}=1 / r_{i}=\frac{4 A}{d_{(i-1) i} d_{i(i+1)} d_{(i-1)(i+1)}}
$$

With the assumption of equidistance edges, and using the fact that the area of a triangle $a b c$ is $A=$ 


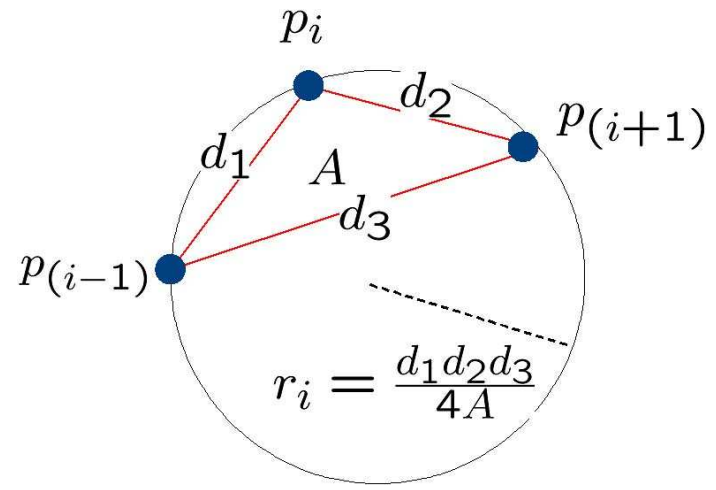

Fig. 1. Approximation of a smooth curve by a polygonal curve

$$
\begin{gathered}
\sqrt{s(s-a)(s-b)(s-c)} \text {, where } s=\frac{a+b+c}{2} \text {. we get } \\
\kappa_{i}=\frac{2 \sqrt{d^{2}-\frac{d_{(i-1)(i+1)}^{2}}{4}}}{d^{2}} .
\end{gathered}
$$

Since $\gamma$ has a maximum curvature $\kappa$; therefore, $\kappa_{i} \leq \kappa$, which implies

$$
\left\|p_{i-1}-p_{i+1}\right\|=d_{(i-1)(i+1)} \geq L / n \sqrt{4-\frac{\kappa^{2} L^{2}}{n^{2}}}
$$

In summary, the $n+1$ ordered points $p_{0}, p_{1}, \ldots, p_{n} \in \mathbb{R}^{2}$, where $p_{0}=P, p_{n}=Q$, form an equidistance polygonal curve with maximum curvature $\kappa$ and length $L$, if and only if, for all $i=1 \ldots n$, distances $\left\|p_{i-1}-p_{i}\right\|$ and $\left\|p_{i-1}-p_{i+1}\right\|$ satisfy (2) and (5).

One way of finding points $p_{0}, p_{1}, \ldots, p_{n} \in \mathbb{R}^{2}$, that satisfy constraints (2), and (5) is modeling the problem as feasibility Problem as follows.

$$
\begin{aligned}
& \min _{\left\{p_{1}, \ldots, p_{n-1}\right\} \in \mathbb{R}^{2}} 0 \\
& \text { s.t.: } \\
& \quad p_{0}=P \quad \text { and } \quad p_{n}=Q, \\
& \quad\left\|p_{i}-p_{i-1}\right\|=d, \quad i=1, \ldots, n \\
& \left\|p_{i-1}-p_{i+1}\right\| \geq \eta, \quad i=1, \ldots, n-1
\end{aligned}
$$

The optimization problem (6) is a nonconvex problem. In the following section, we we propose a multi-particle dynamical system approach to solve the feasibility problem (6). First, we consider the path planning problem without obstacles.

\section{Path Planning Using Stable Multi-Particle SYSTEMS}

\section{A. Continuous Forces}

In this section, we propose a method to find a feasible solution of problem (6) for a single vehicle in the absence of obstacles in the environment.

Consider the waypoints of the vehicle as $p_{0}, \ldots, p_{n} \in \mathbb{R}^{2}$. Now lets assume that the points $p_{0}, \ldots, p_{n} \in \mathbb{R}^{2}$ are point mass moving particles with initial random distribution in the space. Also assume that the vector $F_{i}$ is a force acting on the $i-t h$ point. Therefore, we have

$$
m_{i} \ddot{p_{i}}=F_{i}
$$

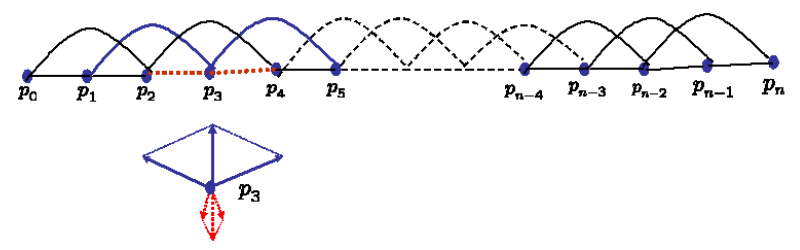

Fig. 2. Analysis of the net forces in the equilibrium which shows that the net forces in $p_{3}$ could be zero even though the forces are not zero)

Now assume $p_{0}=P$ and $p_{n}=Q$ are fixed, we can enforce this condition by assuming $m_{0}, m_{n} \rightarrow \infty$.

In the following we try to find vector forces $F_{i}, i=0 \ldots n$ such that, the set of stable equilibria of the dynamical system (7) would be equal to the set of points $p_{0}, \ldots, p_{n}$ that satisfies constraints (2) and (5).

Define vector forces $F_{i}, i=0 \ldots n$ as follows:

$$
F_{i}=\sum_{\substack{j=0 \\ j \neq i}}^{n} f_{i j}\left(\left\|p_{i}-p_{j}\right\|\right) \mathbf{e}_{i j}-v \dot{p}_{i}
$$

Where $e_{i j}=\frac{p_{j}-p_{i}}{\left\|p_{i}-p_{j}\right\|}$ is a unit vector in the direction of $p_{i}$ to the $p_{j}$.

With the following conditions on functions $f_{i j}$

(i) $f_{i j}=f_{j i}$ for all $i$ and $j$.

(ii) Functions $f_{i j}$ are nondecreasing.

(iii) The vector $\left(p_{0}, \ldots, p_{n}\right)$ is a feasible solution of problem (6) if and only if $f_{i j}\left(\left\|p_{i}-p_{j}\right\|\right)=0$ for all $i, j=0, \ldots, n$.

Theorem 1: All feasible solutions of problem (6) are locally asymptotically stable equilibria of the multi-particle system (7) where $F_{i}$ defined in (8)

Due to space limitation the proof is eliminated. One should note that dynamical system (7) with continuous vector forces (8) may have some additional unfavorable equilibria. A simple analysis shows that in an equilibrium the net force on each particle $p_{i}$ can be zero while some of the force components are not zero (Fig. 2). In fact, nonzero forces in an equilibrium imply infeasibility of the corresponding solution (path). This may arise the possibility of converging to infeasible solutions. In the next section, we will show that by using discontinuous forces such possibilities can be eliminated. It can be proved that all unfavorable equilibria (corresponding to infeasible paths) are unstable. Some additional restrictions on the initial and final orientations of vehicle can be imposed. This can be done by fixing the positions of particles $p_{1}$ and $p_{n-1}$ additional to $p_{0}$ and $p_{n}$ by imposing the constraints $m_{2}, m_{n-1} \rightarrow \infty$.

\section{B. Discontinuous Forces}

In the previous section we tried to convince the reader that by using continuous forces we will always have unfavorable equilibria. In this subsection we use discontinuous forces and by using net force analysis we show that an equilibrium is stable iff all of the forces are zero. Since we are using discontinues forces, we need nonsmooth analysis and stability 
of nonsmooth systems to analyze the dynamical system with discontinuous right-hand sides, but due to space limitation we omit this part. We restrict our discontinuous forces to the following set of functions

$$
f(z)=\left\{\begin{array}{clc}
0 & \text { if } \quad z \geq \eta \\
-w & \text { if } \quad z<\eta
\end{array},\right.
$$

Where $w, \eta \geq 0$ are constant.

Theorem 2: All feasible solutions of problem (6) are locally asymptotically stable equilibria of the multi-particle system (7) where

$$
F_{i}=\sum_{\substack{j=0 \\ j \neq i}}^{n} f_{i j}\left(\left\|p_{i}-p_{j}\right\|\right) \mathbf{e}_{i j}-v \dot{p}_{i} .
$$

Forces $f_{i j}$ 's are either continuous as defined in (8) or discontinuous forces in the form of (9), also $\mathbf{e}_{i j}=\frac{p_{i}-p_{j}}{\left\|p_{i}-p_{j}\right\|}$, and $v>0$ is a constant.

Proof: See appendix for a proof.

Now we define the functions $f_{i j}$ that satisfy the conditions of the theorem (1) as follows

$f_{i(i+1)}(z)=\left\{\begin{array}{ccc}w_{1} & \text { if } & \left(z-\frac{l}{n}\right) \geq \frac{w_{1}}{k_{f}} \\ k_{f}\left(z-\frac{l}{n}\right) & \text { if } & -\frac{w_{1}}{k_{f}} \leq z \leq \frac{w_{1}}{k_{f}} \\ -w_{1} & \text { if } & \left(z-\frac{l}{n}\right) \leq-\frac{w_{1}}{k_{f}}\end{array}\right.$,

and

$$
f_{(i-1)(i+1)}(z)=\left\{\begin{array}{clc}
0 & \text { if } & z \geq \eta \\
-w_{2} & \text { if } & z<\eta
\end{array},\right.
$$

where $\eta=\frac{l}{n} \sqrt{4-\frac{\kappa_{\max }^{2} l^{2}}{n^{2}}}$ and $w_{1}, w_{2}, k_{f}>0$ are some constant numbers. An unfavorable equilibrium is one that the net forces on the particles are equal to zero but some of the forces are not equal to zero. Please note that all of the hard constrains (equidistance and curvature) are satisfied iff all of the forces are equal to zero. In the following theorem we show that with the forces defined in (11), (12) and with the key assumption that $w_{2}>2 w_{1}$ and even number of particles, there would be at most one unfavorable equilibrium which is unstable, therefore for almost all of the initial conditions the dynamical system converges to a favorable equilibrium.

Theorem 3 (Instability of the unfavorable equilibrium): Consider a dynamical system of $2 n$ particles with the forces defined in (11) and (12) also assume that $L>\left\|p_{0}-p_{f}\right\|$ then there is at most one unfavorable equilibrium and and this equilibrium is unstable.

Proof: In the previous theorem we prove that the dynamical system is asymptotically stable so the state of the system converges to a stable equilibrium, in the equilibrium the net force on each particle is zero but not necessary all of the forces are zero. There are two type of forces, the forces that are between consecutive particles $V_{i, i+1}=f_{i, i+1}\left(\| p_{i}-\right.$ $\left.p_{i+1} \|\right) e_{i, i+1}$ for enforcing $\left\|p_{i}-p_{i+1}\right\|=L /(2 n+1)$ and curvature constraint derived forces $V_{i-1, i+1}=f_{i-1, i+1}\left(\| p_{i-1}-\right.$ $\left.p_{i+1} \|\right) e_{i-1, i+1}$. We have

$$
\left\|V_{i, i+1}\right\| \leq w_{1}<w_{2} / 2
$$

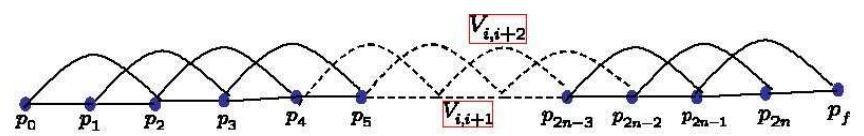

Fig. 3. The nodes represent the particles and the edges represent interaction forces between the particles.

also

$$
\left\|V_{i-1, i+1}\right\|=0 \text { or } w_{2}
$$

Now let construct a graph that nodes of the graph represent the particles and the edges represent the interaction forces (Fig. 3). Any particle except $p_{1}$ and $p_{2 n}$ is connected to 4 other particles (note that $p_{0}$ and $p_{f}$ are not moving particles). At node $p_{1}$ there are 3 forces $V_{0,1}, V_{1,2}$ and $V_{1,3}$, since the net force is zero therefore

$$
V_{0,1}+V_{1,2}+V_{1,3}=0
$$

So

$$
\left\|V_{0,1}+V_{1,2}\right\|=\left\|V_{1,3}\right\|
$$

Now we claim that $V_{1,3}=0$ otherwise we have $V_{1,3}=w_{2}$ so

$$
\left\|V_{0,1}+V_{1,2}\right\|=\left\|V_{1,3}\right\|=w_{2}
$$

But we have

$$
\left\|V_{0,1}+V_{1,2}\right\| \leq\left\|V_{0,1}\right\|+\left\|V_{1,2}\right\| \leq 2 w_{1}<w_{2}
$$

Which is a contradiction, therefore $V_{1,3}=0$. Since the edge $V_{1,3}$ is eliminated now there are 3 edges connected to node $p_{3}$ which are $V_{2,3}, V_{3,4}$ and $V_{3,5}$, with the similar argument one can show that $V_{3,5}=0$, similarly $V_{5,7}=$ $0, \ldots, V_{2 i-1,2 i+1}=0, \ldots, V_{2 n-1, f}=0$. So all of the curvature constraint driven forces connecting odd edges are eliminated. Now one can use the exact same argument this time starting from node $p_{2 n}$, since $p_{2 n}$ connected to 3 nodes, one could conclude $V_{2 n, 2 n-2}=0$ and similarly $V_{2 n-2,2 n-4}=0$ and $V_{2 n-4,2 n-6}=0, \cdots$. Now all of the curvature constraint driven forces are eliminated and the only way that $V_{i, i+1} \neq 0$ with the net force equal to zero is that all of the particles are in a line connecting $p_{0}$ and $p_{f}$ which is clearly unstable.

Please note that if $L \leq\left\|p_{0}-p_{f}\right\|$ there would be just one equilibrium (which is stable) and which is the configuration that all of the particles are in a line connecting $p_{0}$ and $p_{f}$.

\section{Single Vehicle Path Planning with Obstacles}

Let's assume that obstacles are union of disks with centers $o_{i} i=1 \ldots m$ and radii $r_{i} i=1 \ldots m$. In order to fix the location of $o_{i}$, we also assume that $o_{i}$ is an infinite mass moving particles that interact with the waypoints.

Thus, we define obstacle avoidance force between obstacle $o_{i}$ and waypoint $p_{j}$ as

$$
f_{i j}(\alpha)=\left\{\begin{array}{cc}
0 & \text { if }
\end{array} \quad \alpha \geq r_{j} .\right.
$$

Please note that the force defined in Eq. (19) actually satisfies the required conditions in theorem 2 , therefore with obstacle avoidance forces the system still would be asymptotically 


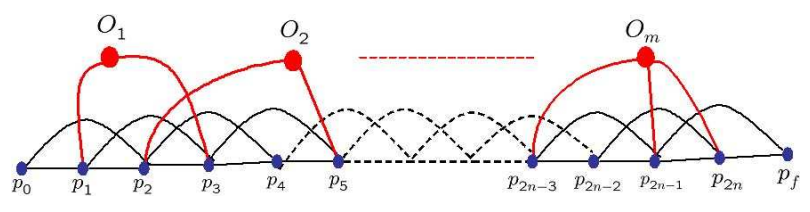

Fig. 4. Graph representation of the forces in the presence of obstacles.

stable.

Based on the obstacle avoidance force definition, there would be a force between a particle and an obstacle iff the particle lies inside of the obstacle, and since the obstacles don't have any overlap at any time each particle could interact with at most one obstacle. Therefore in the connectivity graph each particle could be connected to at most one obstacle. One example of the graph representation of the forces is depicted in fig. (4). In the following theorem we prove that if there exist a solution to the path planning problem then with the assumption of even number of particles and assumption that

$$
2\left(w_{1}+w_{2}\right)<w_{3}
$$

then all of the stable equilibria of the system are actually desirable.

Theorem 4 (Instability of unfavorable equilibria):

Assume that there are $2 n$ particles and obstacles are union of disks with centers $o_{i} i=1 \ldots m$ and radii $r_{i} i=1 \ldots m$ and the forces are defined as Eq. (11), (12) and (20) with the following constraints on $w_{1}, w_{2}$ and $w_{3}$

$$
\begin{gathered}
2 w_{1}<w_{2} \\
2\left(w_{1}+w_{2}\right)<w_{3}
\end{gathered}
$$

then all of the stable equilibria are favorable.

Proof: Fist we prove that in any equilibrium all of the particles are outside of the obstacles. Let assume that $p_{i}$ is inside the obstacle $O_{j}$ therefore $p_{i}$ connected to 5 nodes as follows

- Obstacle avoidance force as $V_{i, j}=w_{3} e_{i, j}$

- Equidistance driven forces as $V_{i-1, i}=f_{i-1, i} e_{i-1, i}$ and $V_{i, i+1}=f_{i, i+1} e_{i, i+1}$

- Curvature forces as $V_{i, i+2}=f_{i, i+2} e_{i, i+2}$ and $V_{i-2, i}=$ $f_{i-2, i} e_{2-1, i}$

Net force on $p_{i}$ is zero so

$$
V_{i-1, i}+V_{i, i+1}+V_{i-2, i}+V_{i, i+2}+V_{i, j}=0
$$

and

$$
\left\|V_{i-1, i}+V_{i, i+1}+V_{i-2, i}+V_{i, i+2}\right\|=\left\|V_{i, j}\right\|=w_{3}
$$

But

$$
\begin{array}{r}
\left\|V_{i-1, i}+V_{i, i+1}+V_{i-2, i}+V_{i, i+2}\right\| \leq \\
\left\|V_{i-1, i}\right\|+\left\|V_{i, i+1}\right\|+\left\|V_{i-2, i}\right\|+\left\|V_{i, i+2}\right\| \leq \\
w_{1}+w_{1}+w_{2}+w_{2}=2\left(w_{1}+w_{2}\right)<w_{3}
\end{array}
$$

Which is contradiction with equality in eq. (23). Therefore in the equilibrium all of particles are outside of the obstacles and there are no forces between particles and obstacles. But in theorem (5) we proved that in this case all of the stable equilibria are favorable.

Figure 5 shows a generated fixed length bounded-curvature trajectory connecting two points and avoiding obstacles.

\section{APPENDIX}

\section{A. Nonsmooth Stability Analysis}

In this section first we review Filipov solution concept for differential equations with discontinuous right-hand sides, the nonsmooth analysis of Clarkes generalized gradient and Lyapunov theorem for nonsmooth systems. We refer the interested reader to the references [10]- [14] for further details.

A function $f$ defined on some topological space $X$ is called locally(essentially) bounded, if for any $x \in X$ there exists a neighborhood $U \subseteq X$ of $x$ such that $f(U)$ is a (essentially) bounded set. The essential supremum is the proper generalization of the maximum to measurable functions, the technical difference is that the values of a function on a set of measure zero don't affect the essential supremum. Given two metric spaces $\left(X, d_{X}\right)$ and $\left(Y, d_{Y}\right)$ the function $f: X \rightarrow Y$ is called locally Lipschitz continuous if for any $x \in X$ there exists a neighborhood $U \subseteq X$ of $x$ so that $f$ restricted to $U$ is Lipschitz continuous. The following are some facts about locally Lipschitz functions

- Any $C^{1}$ function is locally Lipschitz, the proof follows from the mean value theorem and the fact that continuous functions on a locally compact space are bounded.

- Composition of locally Lipschitz functions is locally Lipschitz [15].

- Sum of locally Lipschitz functions is locally Lipschitz.

Lemma 1: A continuous and piecewise differentiable function is locally Lipschitz. The proof is eliminated due to space limitation.

Given function $f: R^{m} \rightarrow R^{n}$, the right directional derivative of $f$ at $x$ in the direction of $v \in R^{m}$ is defined as

$$
f^{\prime}(x ; v)=\lim _{h \rightarrow 0^{+}} \frac{f(x+h v)-f(x)}{h}
$$

On the other hand, the generalized directional derivative of $f$ at $x$ in the direction of $v \in R^{m}$ is defined as

$$
f^{o}(x ; v)=\limsup _{y \rightarrow x} \frac{f(y+h v)-f(y)}{h}
$$

A function $f: R^{m} \rightarrow R^{n}$ is regular at $x \in R^{m}$ if for all $v \in R^{m}$, the right directional derivative of $f$ at $x$ in the direction of $v$ exists, and $f^{\prime}(x ; v)=f^{o}(x ; v)$. The following are some facts about regular functions [10].

- Any $C^{1}$ function is regular.

- A function which can be written as the pointwise maximum of a set of smooth functions is regular.

- Sum of regular functions is also regular.

Now lets consider an autonomous dynamical system as

$$
\dot{x}=f(x, t)
$$

Where $f: R^{n} \times R \rightarrow R^{n}$ is measurable and essentially locally bounded. We must first define what it means to be 


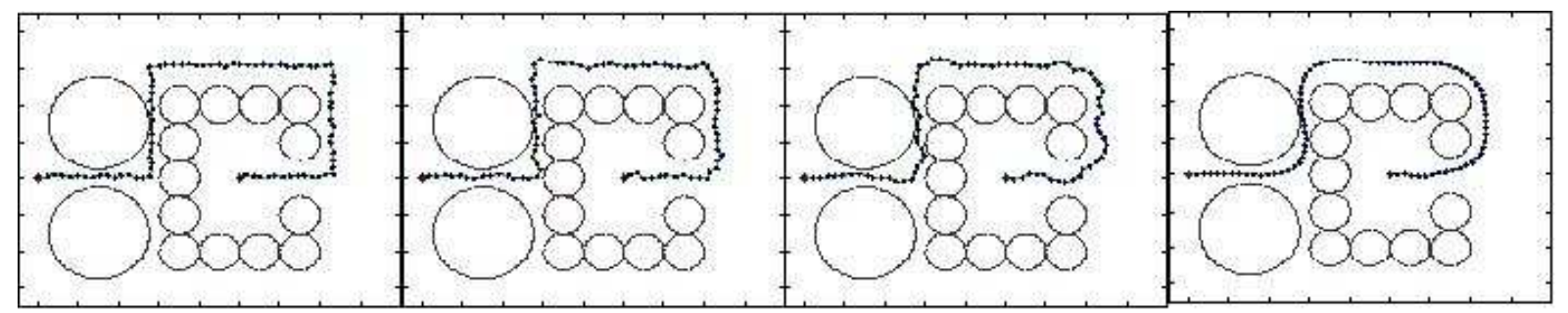

Fig. 5. An example of a fixed length bounded-curvature trajectory connecting two points and avoiding obstacles.

a Filipov solution of this equation. A vector function $x($. is called a solution of (27) on $\left[t_{0}, t_{l}\right]$ if $x($.$) is absolutely$ continuous on $\left[t_{0}, t_{l}\right]$ and for almost all $t \in\left[t_{0}, t_{l}\right]$

$$
\dot{x} \in K[f](x, t)
$$

where

$$
K[f](x, t)=\bigcap_{\delta>0} \bigcap_{\mu N=0} \overline{c o f}(B(x, \delta)-N, t)
$$

and $\bigcap_{\mu N=0}$ denotes the intersection over all sets $\mathrm{N}$ of Lebesgue measure zero. In this formula, $\overline{c o}$ denotes convex closure, and $\mu$ denotes the Lebesgue measure. For a locally Lipschitz function $V: R^{n} \times R \rightarrow R^{n}$ define the Clarke's generalized gradient of $V$ at $(x, t)$ by

$$
\partial V=\overline{c o}\left\{\lim \nabla V(x, t) \mid\left(x_{i}, t_{i}\right) \rightarrow(x, t),\left(x_{i}, t_{i}\right) \notin \Omega_{V}\right\}
$$

where $\Omega_{V}$ is the set of measure zero where the gradient of $\mathrm{V}$ is not defined.

Theorem 5 (Chain Rule [14]): Let $x($.$) be a Filipov solu-$ tion to $\dot{x}=f(x, t)$ on an interval containing $t$ and $V: R^{n} \times$ $R \rightarrow R^{n}$ be a Lipschitz and in addition, regular function. Then $V(x(t), t)$ is absolutely continuous, $(d / d t) V(x(t), t)$ exists almost everywhere and

$$
\frac{d}{d t} V(x, t) \epsilon^{a . e \cdot} \dot{\tilde{V}}(x, t) \equiv \bigcap_{\xi \in \partial V(x(t), t)} \xi^{T}\left[\begin{array}{c}
K[f](x, t) \\
1
\end{array}\right]
$$

Theorem 6 (Nonsmooth Lyapunov theorem [14]): Let $\dot{x}=f(x, t)$ be essentially locally bounded and $0 \in K[f](0)$. Also let $V: R^{n} \times R \rightarrow R$ be a regular function satisfying $V(0)=0$ and

$$
0<V_{1}(\|x\|) \leq V(x) \leq V_{2}(\|x\|)
$$

in a neighborhood of origin for some $V_{1}, V_{2} \in$ class $\mathcal{K}$ [9]. Then $\dot{\tilde{V}}(x, t) \leq 0$ implies $x(t)=0$ is a uniformly stable solution.

Theorem 7: (LaSalle [14]) Let $\Omega$ be a compact set such that every Filipov solution to the autonomous dynamical system $\dot{x}=f(x)$ start in $\Omega$ is unique and remains in $\Omega$. Let $V: \Omega \rightarrow R$ be a time independent regular function such that $v \leq 0$ for all $v \in \dot{\tilde{V}}$, then every trajectory in in $\Omega$ converges to the largest invariant set, $M$, in closure of the set $S=\{x \in \Omega \mid 0 \in \dot{\tilde{V}}\}$.

\section{B. Proof of Theorem 2}

For the dynamical system we define Lyapunov function $E(p, \dot{p})$ as follows

$$
E(p, \dot{p})=\sum_{j=i+1}^{n} \sum_{i=0}^{n-1} W_{i j}\left(\left\|p_{i}-p_{j}\right\|\right)+\frac{1}{2} \sum_{i=0}^{n} m_{i}\left\|\dot{p}_{i}\right\|^{2}
$$

Where

$$
W_{i j}(\alpha)=\int_{\alpha_{0}}^{\alpha} f_{i j}(\xi) d \xi
$$

and $\alpha_{0}$ is root of $f_{i j}$, i.e. $f_{i j}\left(\alpha_{0}\right)=0$. Since function $f_{i j}$ is nondecreasing, $W_{i j}(\alpha) \geq 0$, therefore, $E(p, \dot{p}) \geq 0$. Indeed, before using Nonsmooth Lyapunov theorem we need to show that $E(p, \dot{p})$ is regular and locally Lipschitz function. So we need to show that if $f_{i j}$ is not continuous then $h_{i j}(p)=$ $W_{i j}\left(\left\|p_{i}-p_{j}\right\|\right)$ is regular and locally Lipschitz. Function $h_{i j}(p)$ can be written as the pointwise maximum of a set of smooth(linear) functions so $h_{i j}(p)$ is regular. Also based on lemma 1 function $W_{i j}$ is locally Lipschitz. Function $h_{i j}(p)$ which is composition of two locally Lipschitz functions is locally Lipschitz. Now we can use Nonsmooth Lyapunov theorem. For simplicity we drop $(p, \dot{p})$ from $E(p, \dot{p})$. Also we use $f_{i j}($.$) in replacement of f_{i j}\left(\left\|p_{i}-p_{j}\right\|\right)$.

$$
\dot{\tilde{E}}=\bigcap_{\xi \in \partial E} \xi^{T} K\left[\begin{array}{c}
\sum_{j \neq 1} f_{1 j}(.) \mathbf{e}_{1 j}-v \dot{p_{1}} \\
\vdots \\
\sum_{j \neq n} f_{n j}(.) \mathbf{e}_{n j}-v \dot{p_{n}}
\end{array}\right]
$$

Let's assume that at the point $p$ some of the $f_{i j}\left(\left\|p_{i}-p_{j}\right\|\right)$ are discontinuous. Also let $S$ denotes the set of indices of $f_{i j}$ which are discontinuous at $p$. Let

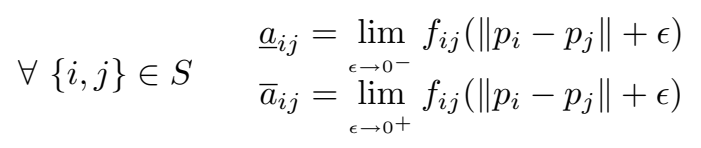

Since functions $f_{i j}$ are nondecreasing $\underline{a}_{i j}<\bar{a}_{i j}$, thus

$$
\partial E=-\left[\begin{array}{c}
\sum_{\{1, j\} \in S}\left[\underline{a}_{1 j}, \bar{a}_{1 j}\right] \mathbf{e}_{1 j}+\sum_{\{1, j\} \notin S} f_{1 j}(.) \mathbf{e}_{1 j} \\
\vdots \\
\sum_{\{n, j\} \in S}\left[\underline{a}_{n j}, \bar{a}_{n j}\right] \mathbf{e}_{n j}+\sum_{\{n, j\} \notin S} f_{n j}(.) \mathbf{e}_{n j}
\end{array}\right]
$$


Also

$$
\left.\begin{array}{c}
K\left[\begin{array}{c}
\dot{p} \\
\sum_{j \neq 1} f_{1 j}(.) \mathbf{e}_{1 j}-v \dot{p_{1}} \\
\vdots \\
\sum_{j \neq n} f_{n j}(.) \mathbf{e}_{n j}-v \dot{p_{n}}
\end{array}\right] \subseteq \\
{\left[\sum_{\{1, j\} \in S}\left[\underline{a}_{1 j}, \bar{a}_{1 j}\right] \mathbf{e}_{1 j}+\sum_{\{1, j\} \notin S} f_{1 j}(.)\right) \mathbf{e}_{1 j}-v \dot{p_{1}}} \\
\left.\sum_{\{n, j\} \in S}\left[\underline{a}_{n j}, \bar{a}_{n j}\right] \mathbf{e}_{n j}+\sum_{\{n, j\} \notin S} f_{n j}(.)\right) \mathbf{e}_{n j}-v \dot{p_{n}}
\end{array}\right]
$$

Let $\xi \in \partial E(p, \dot{p})$, thus

$$
\xi=\left[\begin{array}{c}
-\sum_{\{1, j\} \in S} \xi_{1 j} \mathbf{e}_{1 j}-\sum_{\{1, j\} \notin S} f_{1 j}(.) \mathbf{e}_{1 j} \\
\vdots \\
-\sum_{\{n, j\} \in S} \xi_{n j} \mathbf{e}_{n j}-\sum_{\dot{p}, j\} \notin S} f_{n j}(.) \mathbf{e}_{n j}
\end{array}\right]
$$

Where $\xi_{i j} \in\left[\underline{a}_{i j}, \bar{a}_{i j}\right]$. As a result,

$$
\begin{aligned}
\dot{\tilde{E}}= & \bigcap_{\xi_{i j} \in\left[\underline{a}_{i j}, \bar{a}_{i j}\right]} \sum_{\{i, j\} \in S}\left[\underline{a}_{i j}-\xi_{i j}, \bar{a}_{i j}-\xi_{i j}\right] \dot{p}_{i} \cdot \mathbf{e}_{i j}-v \sum_{i=1}^{n}\left\|\dot{p}_{i}\right\|^{2} \\
\dot{\tilde{E}} & =\sum_{\{i, j\} \in S} \bigcap_{\xi_{i j} \in\left[\underline{a}_{i j}, \bar{a}_{i j}\right]}\left[\underline{a}_{i j}-\xi_{i j}, \bar{a}_{i j}-\xi_{i j}\right] \dot{p}_{i} \cdot \mathbf{e}_{i j} \\
& -v \sum_{i=1}^{n}\left\|\dot{p}_{i}\right\|^{2} \\
& =-v \sum_{i=1}^{n}\left\|\dot{p}_{i}\right\|^{2} \leq 0
\end{aligned}
$$

The last equality is the result of the fact that for any given interval $[a, b]$ we have $\bigcap_{\xi \in[a, b]}[a-\xi, b-\xi]=0$.

This establishes the stability but not the asymptotic stability of favorable equilibria. We use nonsmooth version of LaSalle's theorem to prove locally asymptotically stability of the favorable equilibria. Let $\left(p_{0}, 0\right)$ be a favorable equilibrium of the dynamical system, now consider $N\left(\left(p_{0}, 0\right), \epsilon\right)$ a $\epsilon$-neighborhood of the favorable equilibrium such that there is no unfavorable equilibrium in $N\left(\left(p_{0}, 0\right), \epsilon\right)$. Now let $\Omega=$ $\{(p, \dot{p}) \mid E(p, \dot{p}) \leq 1\} \bigcap N\left(\left(p_{0}, 0\right), \epsilon\right)$. As a consequence of LaSalle (Theorem 7), the trajectory enters the largest invariant set in

$$
\Omega \bigcap\{(p, \dot{p}) \mid 0 \in \dot{\tilde{E}}\}=\Omega \bigcap\{(p, \dot{p}) \mid \dot{p}=0\}
$$

To obtain the largest invariant set in this region note that $\dot{p}=$ 0 and $p=$ constant, as a result the trajectory converges to an equilibrium. But in $\Omega$ there is no unfavorable equilibrium, consequently the manifold of favorable equilibria is locally asymptotically stable.

\section{REFERENCES}

[1] A. Ahmadzadeh, A. Jadbabaie, G. J. Pappas, and V. Kumar, Stable multi-particle systems and application in multi-vehicle path planning and coverage, IEEE CDC, New Orleans, December 2007.

[2] J. C. Latombe. Robot Motion Planning. Kluwer Academic Publishers, 1991

[3] L. E. Dubins, On curves of minimal length with a constraint on average curvature, and with prescribed initial and terminal positions and tangents, Amer. J. Math., vol. 79, no. 3, pp. 497-516, 1957

[4] J. Reif and H. Wang. The complexity of the two dimensional curvature-constrained shortestpath problem. In WAFR 98: Proceedings of the third workshop on the algorithmic foundations of robotics on Robotics, Natick, MA, USA, 1998

[5] S. Fortune and G. Wilfong. Planning constrained motion. Ann. Math. Artificial Intelligence, 3(1):2182, 1991

[6] P. Jacobs and J. Canny. Planning smooth paths for mobile robots. In Proceedings of the IEEE International Conference on Robotics and Automation, 1989.

[7] H. Wang and P. K. Agarwal. Approximation algorithms for curvature-constrained shortest paths. In Proceedings of the seventh annual ACM-SIAM symposium on Discrete algorithms, Philadelphia, PA, USA, 1996.

[8] D. E. Koditschek and E. Rimon, Robot navigation functions on manifolds with boundary, Advances Appl. Math., vol. 11, 1990.

[9] S. Sastry, Nonlinear Systems , Springer, 2004.

[10] F.H. Clarke, Yu.S. Ledyaev, R.J. Stern, and P.R. Wolenski. Nonsmooth Analysis and Control Theory. Graduate Texts in Mathematics; 178. Springer, New York, 1998.

[11] A. F. Filipov, Differential equations with discontinuous right-hand side, Amer. Math. Soc. Translations, vol. 42, no. 2, pp. 191-231, 1964.

[12] A. F. Filippov. Differential equations with discontinuous right-hand side. Mathematics and Its Applications (Soviet Series). Kluwer Academic Publishers, The Netherlands, 1988.

[13] J. Corts, Discontinuous dynamical systems - a tutorial on solutions, nonsmooth analysis, and stability IEEE Control Systems Magazine, 2008 , to appear.

[14] D. Shevitz and B. Paden. Lyapunov stability theory of nonsmooth systems. IEEE Transactions on Automatic Control, 39(9), 1994.

[15] I. Gohberg, P. Lancaster and L. RodmanInvariant, Subspaces of Matrices with Applications, SIAM Classics in Applied Mathematics, 2006. 